\title{
TOWARDS REALIZATION OF A HIGH-FIDELITY SIMULATION MODEL FOR SHORT- TERM HORIZON FORECASTING IN WAFER FABRICATION FACILITIES
}

\author{
Wolfgang Scholl \\ Infineon Technologies Dresden \\ Königsbrücker Strasse 180 \\ 01099 Dresden, GERMANY \\ Daniel Noack \\ Oliver Rose \\ Institute of Applied Computer Science \\ Dresden University of Technology \\ 01062 Dresden, GERMANY
}

\author{
Boon Ping Gan \\ Ming Li Peh \\ Peter Lendermann \\ D-SIMLAB Technologies Pte Ltd \\ 9 Jurong Town Hall Road \#03-45 iHUB \\ Singapore 609431, SINGAPORE \\ Patrick Preuss \\ D-SIMLAB Technologies GmbH \\ Manfred-von-Ardenne Ring 20 \\ 01099 Dresden, GERMANY
}

\begin{abstract}
Discrete Event Simulation (DES) has widely been used for mid and long term forecasting in wafer fabrication plants. But the use of DES for short term forecasting has been limited due to the perceived modelling and computation complexity as well as the non-steady state nature of today's wafer fab operations. In this paper, we discuss some important modelling issues associated with building an online simulation model. Key elements considered are actual process routes, process and throughput modelling as a function of equipment behavior, lot size, and available processing modules, process dedication at equipment level, equipment downs at mainframe level, estimated lot release strategy, send ahead wafers, dispatch rules, and setup. Typical application areas are proactive dedication management, preventive maintenance scheduling and WIP based sampling optimization.
\end{abstract}

\section{INTRODUCTION}

At Infineon's semiconductor production line in Dresden, Discrete Event Simulation (DES) using the commercial software AutoSched AP has become a frequently-used decision-making tool (Scholl 2008). Currently, the most important application is medium-term forecasting of key performance indicators for a time horizon of 3 months. But there is also a more pressing need for higher-fidelity short-term forecasting for proactive fab operations management. This need is primarily driven by uncertainty in market demand for semiconductor products that causes disturbances such as WIP waves to the fab operation. If such a disturbance is not well managed, the fab operations will become very volatile. Unpredictability typically results in increase of production cost and this affects a company's competitiveness in the global market place.

The dynamism in market demand requires not only a flexible product flow control but also the ability to adjust fab operation parameters such as dispatch policies. The implication of adjusting fab operation parameters to long-term side effects on other products and overall fab performance has to be examined 


\section{Scholl, Gan, Noack, Preuss, Peh, Lendermann, and Rose}

and managed as well. This effect, however, cannot be sufficiently analysed and understood through real time data analysis and personnel experiences. Rather, the assessment has to be done through short term simulation (also known as online simulation, see Bagchi 2008). Online simulation provides the required visibility into the dynamics of fab operations and the possibility of evaluating alternative countermeasures to appropriately manage these dynamics. Examples of such countermeasures are adjustment of the wafer start plan, process dedication, preventive maintenance schedule and Real Time Dispatcher (RTD) parameters.

Online simulation is transient in nature. It poses different technical challenges as compared to $\mathrm{mid} /$ long term simulation (also known as offline simulation), particularly with regard to model granularity. In an offline simulation model, planning data is used to feed the model. It works with representative routes, "stable" tool dedication in critical production areas such as lithography and sputtering, crucial dispatch rules at fab level, and simple equipment configuration modelling. Typically, the execution time of offline simulation (with 3 months time horizon) is 12 hours for 10 confidence runs. In contrast, an online simulation model is fed with the current fab state for warm-starting and detailed fab data for fine grain modelling. It works with actual routes, tool dedication in all production areas, dispatch rules at work centre level, and detailed equipment configuration modelling. Typical execution time of online simulation (with 3 days time horizon) is less than an hour for 10 confidence runs. An alternative approach to online simulation would be to use a 24-hour prediction tool that is based on planned cycle times. This approach is not sufficient as it is a static approximation, and dynamic situations such as fab loading, tool availability, existence of optional process steps are not considered. Furthermore, the prediction horizon of 24 hours is too short to generate a substantial benefit. The expected benefit of a solid prediction tool must offer at least $2 \%$ to $5 \%$ cycle time variability reduction within the production process, and online simulation bears the most potential.

One example of online simulation application is for engineering and preventive maintenance scheduling. Today, decisions on scheduling of engineering and preventive maintenance events are made based on the actual WIP situation in combination with personnel long-term experiences. Preventive maintenances can thus only be shifted to the end of the restricted time window in case of a high WIP level. With online simulation, preventive maintenance scheduling can be managed more flexibly. The maintenance event can be shifted to an earlier or a later time, depending on the forecasted WIP situation.

Another application of online simulation is pre-emptive resource (such as tools, operators, probe cards in wafer test) releases. Typically, release of additional tools/resources can only become operative 1 to 3 days after occurrence of a critical situation. When this critical situation is forecasted, the release of additional tools/resources will be just-in-time to manage the occurrence of this situation.

Lastly, online simulation can also add significant value when it comes to adjusting sampling rates at measurement steps. Usually, the sampling rate of measurement steps would be adjusted in case of high lot arrival rates. This requires at least half a day to install the modified programs which would have been too late as WIP might have already built up after half day of delay. With online simulation, lot arrival rates can be forecasted and adjustment of sampling rate at measurement steps can be triggered to pre-empt and manage the high lot arrival rate situation before it occurs.

These examples of proactive fab operations management promise tremendous benefit for Infineon's fab operations. In this setting, Infineon Dresden embarked on a joint project with D-SIMLAB and the Technical University of Dresden to realize a live-data based online simulation. In this paper, we present the results of our feasibility study project.

One focus was the development of a data model prototype that gathers data from Infineon's existing data sources. The integration of different data sources with varying degree of data quality was a huge challenge. Missing or incorrect data had to be replaced with historical data. Concurrent to the data model prototype development, we also built a new short term simulation model of higher granularity. It is warmstarted with current fab state, and contains all products, process flows, both fixed and temporary tool dedications, logistics restrictions such as KANBAN rules for process sequences with time-windows, and more detailed equipment modelling that was realized by considering internal material flow and by assign- 
ing equipments to one out of five different equipment classes. With this classification, we were able to realize lot-size dependent throughput and processing time modelling. Other features such as setup, rework and send-ahead wafer procedures were also modelled.

\section{ONLINE SIMULATION MODEL FEATURES}

Online simulation requires a high granularity model in order to achieve a good performance measure forecast accuracy at product and work center level. However, high granularity also means high computational complexity that results in long simulation execution times. Striking a balance between forecast accuracy and computation complexity is thus crucial when an online simulation model is built. In this section, we discuss five important modelling features of our online simulation model, namely lot release strategy, fab current state modelling, equipment modelling (processing time, throughput, dedication, setup, tool down and dispatch rules), process routes and sampling process (step percent and send ahead wafers).

\subsection{Lot Release Strategy}

In Infineon, lot release plays a role not only at frontend-of-line but also backend-of-line. For frontend-ofline release, a fixed number of wafers are released daily into the production line, and releases are defined through a lot release plan. However, this release plan does not define the exact point in time at which lots are to be released. To avoid sudden bursts of releases into the production line, which could potentially create a (small) WIP wave, we staggered the lot releases equally in an 18-hours time span. In contrast, there is no plan available for backend-of-line releases. The only data available is the number of wafers per product group currently in the master storage (a place to hold lots that have completed frontend-of-line processes). With this information, we staggered the lot releases to the backend-of-line over a 7-day time period. Similarly, this approach avoids a sudden burst of releases that potentially could create a WIP wave. This granularity of modelling appears to be sufficient as the application areas of online simulation is for a 3-day forecast.

\subsection{Fab Current State Modelling}

An online simulation model also has to be warm-started with the current fab state. This includes (i) WIP waiting to be processed at work center, (ii) WIP currently in process in equipment, including equipment name and start time of lots in the equipment, (iii) lot attributes such as lot identifier, current operation, priority, cumulated cycle time and lot size, and (iv) equipments that are currently down, including the start time of the down event. Two of the key challenges to warm start the online simulation model is the estimation of remaining processing time of WIPs running in equipments and estimated down duration for down equipments. The remaining processing time of WIPs can be estimated when the start time, current operation, and process definition (EPA) of WIP are available. It was more challenging to estimate down duration as the duration depends on the actual issue that triggered the down event. The duration of preventive maintenance down has higher predictability compared to ad-hoc down. Our source of information is through historical data analysis.

\subsection{Equipment Modelling}

Equipment modelling is a very important aspect of online simulation. Processing time and throughput are modelled through analysis of various equipment internal material flows. Our analysis resulted in five different equipment classes that were derived as summarized in Table 1 below. Each equipment in the fab was mapped to one class. For example, wet benches with multiple baths/sinks are classified as equipment model class 2, chemical-mechanical-polisher equipments with sequence of polishing are classified as equipment model class 4 , batch furnaces are classified as equipment model class 1 . During a simulation, the processing times and throughputs of equipment are computed dynamically depending on the equip- 
ment model classes whenever a lot or batch of lots is scheduled. To facilitate this modelling, we need raw processing time and throughput data for all equipments and processes in the fab.

Table 1: Equipment Model Classes

\begin{tabular}{|c|l|}
\hline $\begin{array}{c}\text { Equipment Model } \\
\text { Classes }\end{array}$ & \multicolumn{1}{c|}{ Description } \\
\hline 1 & $\begin{array}{l}\text { Equipments that process lot or batch with one or multiple process modules, } \\
\text { where processing time/throughput is independent of lot or batch size. }\end{array}$ \\
\hline 2 & $\begin{array}{l}\text { Equipments that process lot or batch in a sequence of sub process steps with } \\
\text { one or multiple process modules, where processing time/throughput is indepen- } \\
\text { dent of lot or batch size. }\end{array}$ \\
\hline 3 & $\begin{array}{l}\text { Equipments that process single/multiple wafers with one or multiple process } \\
\text { modules, where processing time/throughput is a function of lot size and number } \\
\text { of available processing modules. }\end{array}$ \\
\hline 4 & $\begin{array}{l}\text { Equipments that process single wafer in a sequence of sub process steps. Proc- } \\
\text { essing time/throughput is a function of lot size. }\end{array}$ \\
\hline 5 & $\begin{array}{l}\text { Equipments that process multiple wafers concurrently, with/without dummy } \\
\text { wafers, where processing time/throughput is a function of lot size, number of } \\
\text { available processing modules, and ability to mix lots. }\end{array}$ \\
\hline
\end{tabular}

Typically, equipments in the fab are qualified to run certain processes that are uniquely identified by process definition (EPA). This is termed as process dedication. Process dedication affects the capacity of the equipments. It is thus crucial to take this into consideration for our online simulation model. In addition, different subsets of these dedications are active at different period of time. The online simulation model has to be initialized with the right dedication subset in order to model the capacity accurately.

Another important modelling consideration for our online simulation model is setup. Setup time is incurred whenever an equipment needs to be re-configured to run a different process. During setup, the equipment is not available for production. This reduces the productive capacity of the respective equipment. In our online simulation model, setup is modelled for lithography and implantation.

In addition to setup changes, down events also reduce the productive capacity of equipments. In general, equipment downs can be classified into two subclasses. They are preventive maintenance and ad-hoc downs. Preventive maintenance (PM) of equipments is typically executed according to a plan, considering the load situation. We incorporated the PM plan into our online simulation model without considering the possibility of deviations. This is because the deviation from plan is typically small and its effect on online simulation is expected to be minimal. One key challenge in equipment down modelling, however, is adhoc down as it is random. The solution to this problem is through analysis of historical data to derive the down interval, duration, and statistical distribution. Another important point to note is that - since we do not model material movement of individual processing chambers (for example cluster tools) - portraying chamber downs becomes a challenge. Our proposed solution to this problem is to abstract chamber downs to equipment downs, but proportionally decrease the down duration. With this approach, we ensure that the equipment productive capacity is reasonably well represented.

The last modelling consideration for equipment model are dispatch rules. We modelled fab level dispatch policies such as lot/step priority, target cycle time, $x$-hour maximum lot waiting time and work center level dispatch policy such as time-window sensitive dispatching. To portray these dispatch rules, we need lot attribute data such as lot priority, cumulated cycle time and waiting time of lots up to current time and all time-window sensitive process steps (including the time window value).

\subsection{Process Routes}

Actual process routes are used in our online simulation model. Each product type is mapped to a unique process route in fab operations. Key attributes of process routes are route name, step name, work center, 


\section{Scholl, Gan, Noack, Preuss, Peh, Lendermann, and Rose}

EPA, batching criteria, recipe, and remaining target cycle time associated with each step. Two important modelling considerations for actual process routes are alternative process steps and sum process steps. Alternative process step is used to model alternative process steps execution based on lot attributes or alternative work center for the process step. Effectively, there is no modelling distinction between them although there is a challenge to model the sum process steps. A sum process step is a collection of multiple sub process steps. Whether a lot takes a sub process step is based on lot attributes or an on-the-fly decision. We are not able to model on-the-fly decision-making for sub process step execution. Instead, we model this using step percent for each sub process step. Step percent defines the percentage of lots that are going to execute the sub process step and can be derived from historical data analysis.

\subsection{Sampling Process Modelling}

Two types of sampling processes, namely step percent and send-ahead wafers are modelled in our online simulation model. Send-ahead wafers is a process in the lithography area where a lot is split into two sublots under certain conditions. One sub-lot contains a small number of wafers while another sub-lot contains the remaining wafers. The smaller sub-lot will proceed through several production steps. Once it completes these production steps without any process issues (typically confirmed after some measurement steps), the larger sub-lot is triggered to run through the same production steps. The two sub-lots are merged back to form a single lot once the larger sub-lot reaches the same process step as the smaller sublot. We incorporated this modelling feature by defining split rate, production step for splitting, and production step for merging. The split rate can be derived from historical data analysis.

\section{DATA REQUIREMENTS}

The online simulation model has to be instantiated with fab data from many data sources. However, retrieving data from multiple data sources during instantiation is rather inefficient. To resolve this inefficiency problem, we created a data model that consolidates data from various data sources and serves as a single point of access for online simulation model instantiation. As our online simulation model will be used for daily forecasting, the data model initiates a query to all relevant data sources once per day. With this approach, the instantiated online simulation model is outdated by at most 8 hours (as we do not expect forecast runs late in the evening). A key requirement of the data model is that the data must be of good quality (consistent, error free, and complete) and reproducible for validation and verification purpose. Detailed discussion on the data model and associated issues can be found in Noack et al. (2010).

\subsection{Processing Time and Throughput}

Multiple data sources are available for processing times and throughput data. Planning data are available from each department or for the whole fab. Other data sources are based on historical analysis for individual equipments and processes. Data sources have been created for different purposes, so they differ in their level of detail. Also, in some cases the timing values contradict with each other among different data sources. This is primarily due to the fact that the definition of processing time and throughput can be different in different data sources. For example, one database might already consider elements such as setup, another might not. Another reason is that equipments with high processing time variability also have different values in different data sources. To solve this problem we compared different processing time values in different data sources. For the feasibility study we selected the data source that matched best with our simulation in terms of level of detail and processing time definition.

\subsection{Dedication}

Equipment dedication has two elements. The first element defines which process can run on which equipment. This information is available with good quality in a single data source. The second element defines several blocking mechanisms, where some processes are temporarily blocked at some equipments. 
This information is distributed and maintained in several production departments. Blocking applies with different levels of detail: Work center/equipment/chamber level or product group/product/lot level. The blocking mechanism needs to be highly flexible to apply changes very fast. However, it is hard to capture blocking information because of the flexible and distributed character. To solve this problem, we need to incorporate the temporary blocking of processes to the data model. This still poses a challenge as this information is sometimes not available from the respective data source but is incorporated into dispatch rules. For the purpose of our feasibility study, we derived dedication information through historical data analysis.

\subsection{Equipment Down}

As discussed earlier, we distinguish preventive maintenance (scheduled) and ad-hoc (unscheduled) downs. As unscheduled downs cannot be predicted, there is no data source available with this information. We recycle the downtime and repair time distribution from our offline simulation model which had been generated from historical data analysis. In contrary, two solutions are feasible for scheduled downs, either by using historical analysis or by using preventive maintenance (PM) plan data. We decided to use the PM plan data because the PM start time is much more accurate compared to the stochastic distribution from historical analysis. The integration of the PM plan requires substantial effort because this information was distributed across heterogeneous data sources in each department.

\subsection{Setup}

For setup we realized that a highly detailed automated approach is not feasible as the number of setup permutations is too high. To resolve this problem, we employed a simple approach by analyzing historical data to generate the setup matrix. The setup matrix is recipe-based, which significantly reduces the number of permutations. This setup matrix is kept as a static data as setup times typically do not change very often.

\subsection{Sampling Data}

We also need to integrate sampling and send-ahead wafers split rate into our online simulation model. This information does not exist on an aggregated level. We therefore used historical analysis to compute sampling and send ahead rates from history lot trace.

\subsection{Initialization Data}

Figure 1 gives an illustration of the data model schema. It contains the most important information from the simulation perspective. The data model contains lot, product, route, equipment, and process and downtime information. As can be seen, WIP lot has detailed information about its current operation, current equipment and remaining processing time. Every equipment also has information about the current equipment state.

\section{ONLINE SIMULATION MODEL VALIDATION}

To validate the simulation model we used a fixed one week period in 2009 which was chosen because less abnormal fab behavior was observed during this time. For all key performance indicators (KPI) we were able to make comparisons between the simulation model and reality. We computed the reality KPI by using the fab lot trace file, where movement events for all lots are being captured (arrival and departure time at an operation, equipment allocated for the lot, etc). This lot trace file also served as a reference for validating the data that feed our online simulation model. The model validation exercise was carried out with the focus as summarized in Table 2 . 


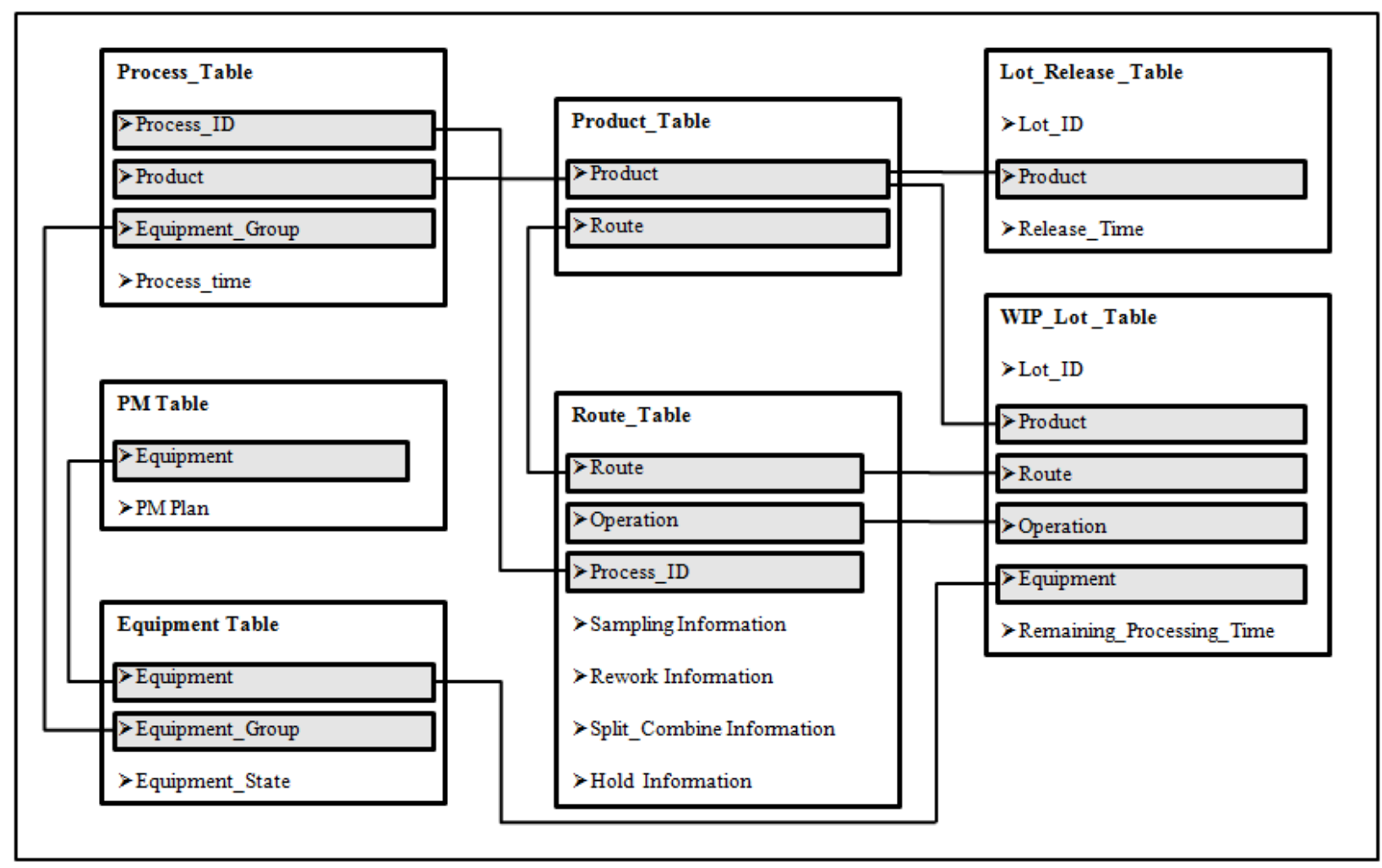

Figure 1: Data Model Schema Overview

Table 2: The Focus Area of Model Validation

\begin{tabular}{|l|l|}
\hline \multicolumn{1}{|c|}{ Analysis } & \multicolumn{1}{c|}{ Descriptions } \\
\hline Initialization & $\begin{array}{l}\text { To measure the deviation of initial WIP at all work center between reality and simula- } \\
\text { tion. The deviation must be small as an inaccurately initialized model is not useable for } \\
\text { forecasting. }\end{array}$ \\
\hline Work center & $\begin{array}{l}\text { To validate the WIP trend, arrival and departure rate at all work centers. The model must } \\
\text { be able to show close proximity between simulated and real trend/values. }\end{array}$ \\
\hline Product & $\begin{array}{l}\text { To validate the wafer out per product type. The model must be able to show good wafer } \\
\text { out forecast at product type level. }\end{array}$ \\
\hline
\end{tabular}

\subsection{Initialization Analysis}

Analysis of model initialization (Reijers and Aalst 1999) includes three aspects: (i) All waiting lots must be assigned to the correct work center, (ii) all running lots have to be assigned to the correct equipment with the correct remaining processing time, and (iii) all down equipments must be initialized as down state. Correct assignment of lots to work center and equipment can be verified by comparing the WIP differences between reality and simulation at all work centers. Figure 2 below gives an overview of this comparison. As can be seen, there is no deviation between reality and simulation for majority of the work centers. The primary reason for discrepancy that is observed for some work centers is the difference in equipment to work center mapping between simulation and reality. In fact, the WIPs are being initialized at the right operation points in the fab. 


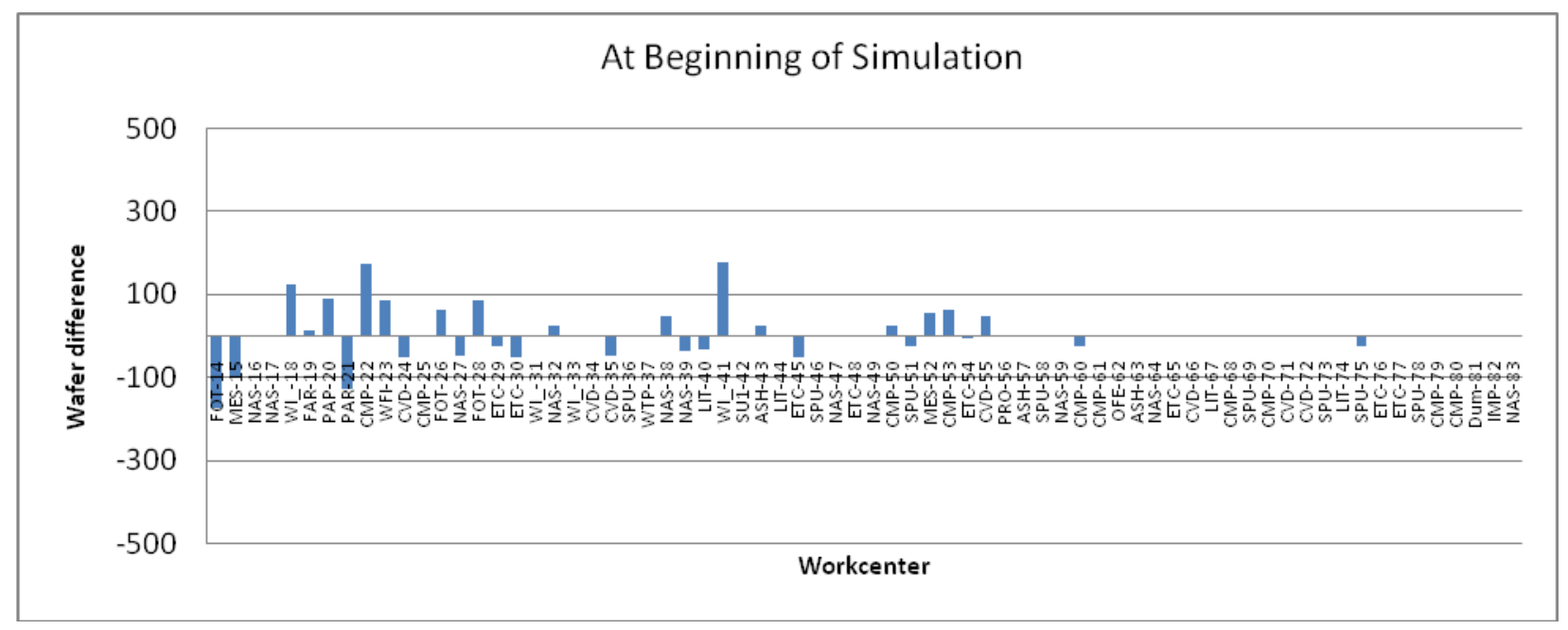

Figure 2: WIP Level Differences for all Work Centers at Initialization

Whether the online simulation model is initialized with correct remaining processing time or down state is validated through the work center analysis described in Section 4.2. We compared the WIP trend of all work centers and observed that the WIP trend for more than $80 \%$ of the work centers does resemble the reality.

\subsection{Work Center Analysis}

In the work center analysis, we compared the WIP level, arrival and departure wafers for all work centers. As there are around 200 work centers in the fab, we are showing only two of the work centers in Figure 3 and 4 . The performance measure is taken at 12 am every day. WIP level is the number of wafers waiting at the work center at that instance of time, wafers arrival/departure is the number of wafers arrived/departed from $12 \mathrm{am}$ of the previous day up to $12 \mathrm{am}$ of current day. As can be seen, our online simulation model not only is able to forecast the trend with reasonable accuracy but also is capable of forecasting the absolute value of WIP at a particular instance of time. During the process of validation, we realized the importance of modelling measurement work center with greater granularity to further improve the forecast accuracy for some work centers.

\subsection{Product Analysis}

An important application of our online simulation model is to forecast the wafer out per product for better wafer test planning. Figure 5 shows a comparison of cumulated wafer out comparison between reality and simulation for two products. These products are chosen because they are high volume products of the fab. As can be seen, our online simulation model is able to forecast wafer out with reasonable accuracy. It shows good trend and absolute value forecast. But further work still need to be performed for product level wafer out forecast. We observed that wafer out for some product type deviates significantly from reality. Two contributing factors of this discrepancy are the local dispatch policies at work center level and abstracted measurement equipment modelling. One of the focus areas in our future work is to resolve these two issues.

\section{SUMMARY AND CONCLUSIONS}

In this paper, we presented our experience in building an online simulation model. We discussed the important modelling elements that must be considered in order to achieve a good forecast accuracy (for a 3 day time horizon). It is interesting to note that an abstracted equipment model of processing time/throughput, dedication, down, and setup is sufficient to achieve a very good work center level fore- 
cast. There is no need to model the internal material flow (such as chamber level modelling) to get a good work center forecast accuracy. This helps to reduce the simulation execution time significantly. Another important point to note is that chamber down being abstracted to equipment level down (with reduced down duration) is also sufficient to give good work center forecast accuracy. This significantly reduces the data granularity required to model chamber level down.

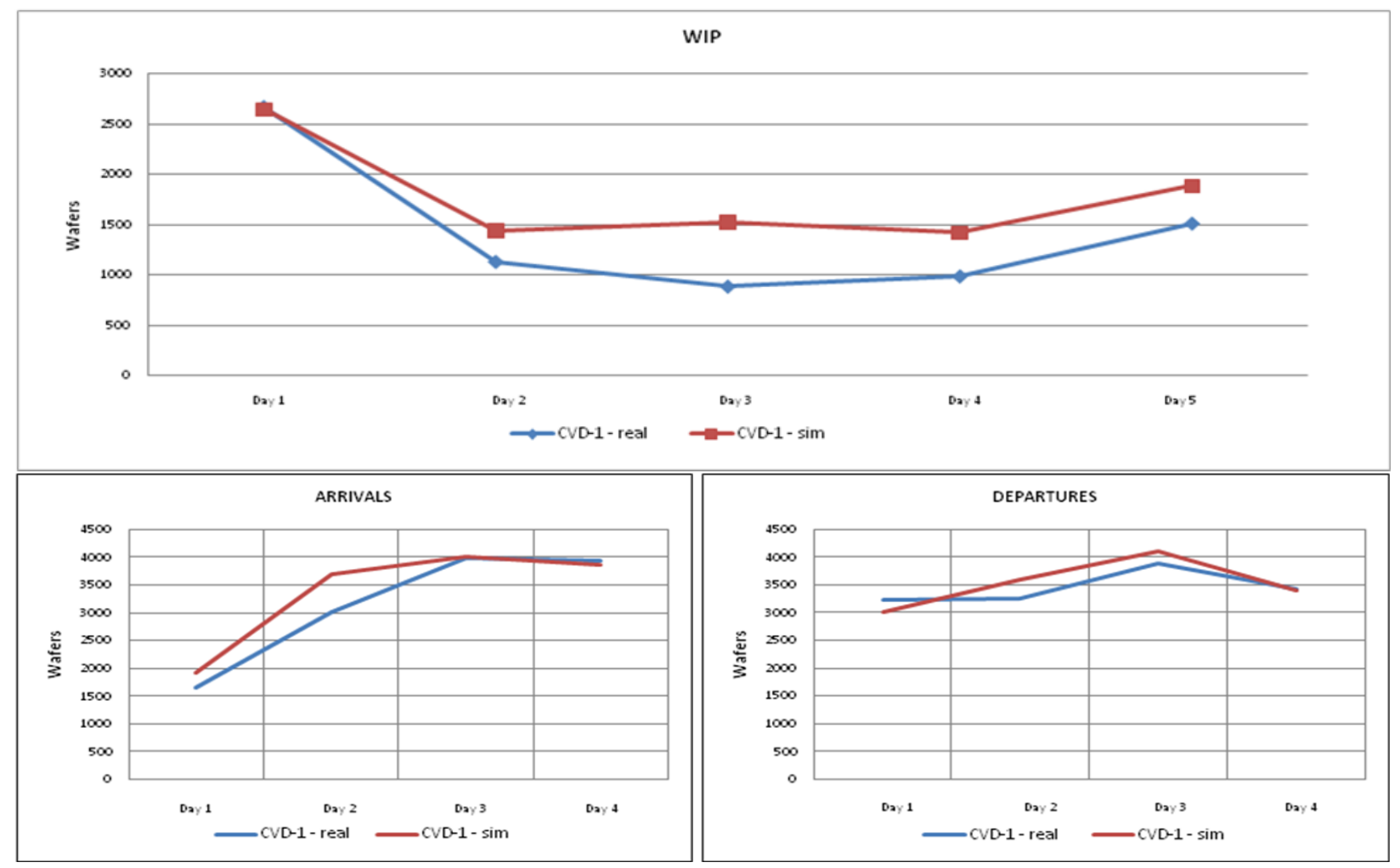

Figure 3: Trend Comparison between Simulation and Reality for a CVD-Cluster Tool

Overall, the model validation results for our online simulation model have been encouraging. We are able to forecast both WIP trends and absolute values at work center level accurately, with minimal discrepancy from reality. The significance of these results is that now we are able to anticipate not only problems at particular work centers but also on which day these problems will arise. This visibility is very useful as it allows to manage fab operations more proactively instead of reactively. The longest time horizon to pre-empt the problem is 3 days, according to production engineers this is considered sufficient. In addition to a good work center level forecast, we are also able to achieve a good wafer out forecast at product level. This greatly facilitates the planning activities of the wafer test area, which now is operating without knowing in advance what wafer out to be expected in the next days.

Though we have observed good forecast accuracy with our online simulation model, there are still several areas of improvement that we need to work on. Firstly, we need to model work center local dispatch policies with much higher granularity. The current approach of fab level dispatch policies modelling is not sufficient to give good forecast accuracy for all product types. Secondly, we need to model the measurement equipments with higher granularity. Currently they are modelled as fixed delays, where the delay is the typical cycle time at the measurement step. The departure rate from these measurement equipments is thus not accurately reflecting the reality. Lastly, we are going to improve the data quality for process dedication to equipments. The current approach of analyzing historical data to derive process dedication is not sufficient as temporary blocking of processes at equipments does not mean the same blocking of processes applies in the next few days. 


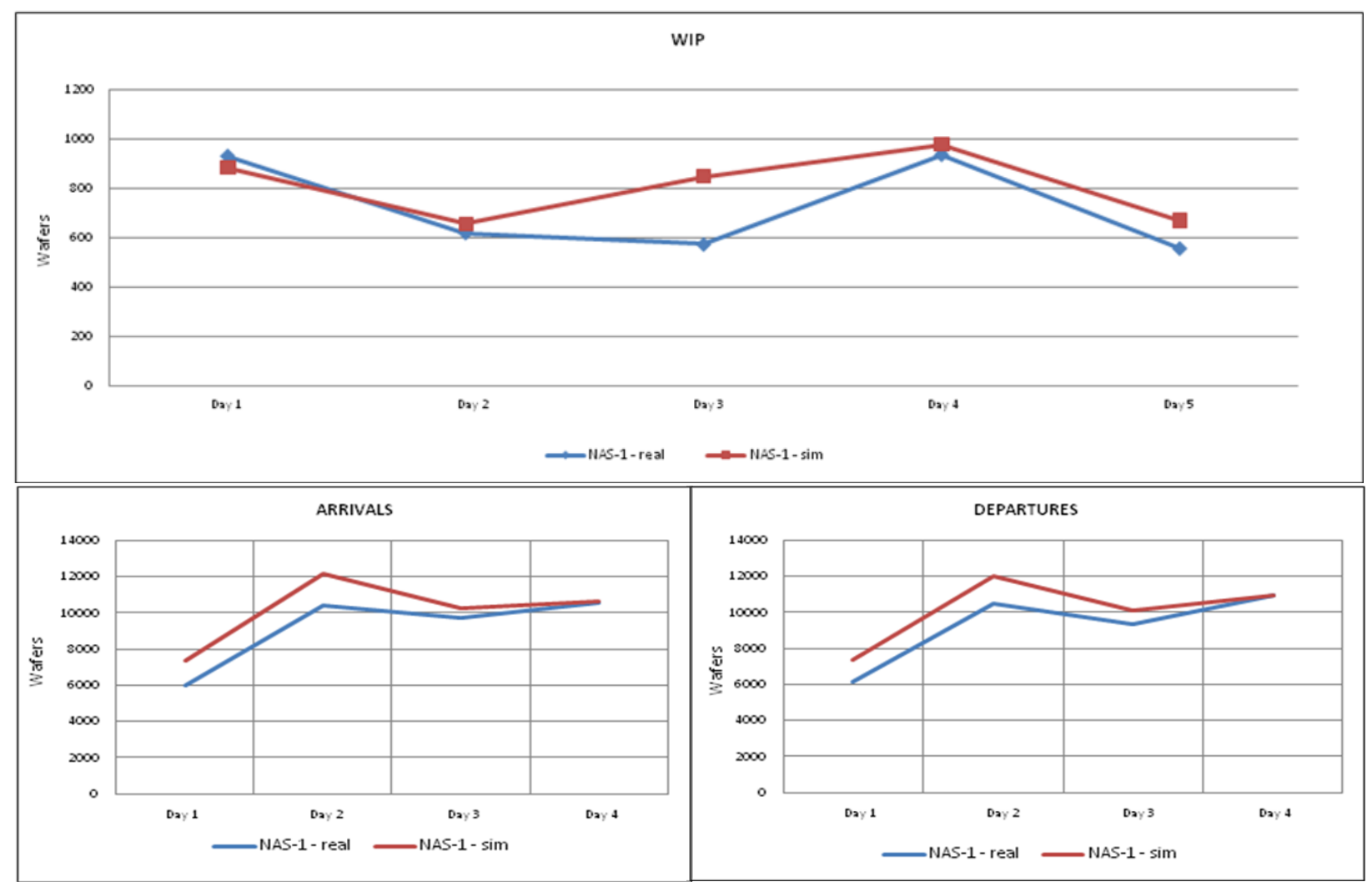

Figure 4: Trend Comparison between Simulation and Reality for Lithography Tool
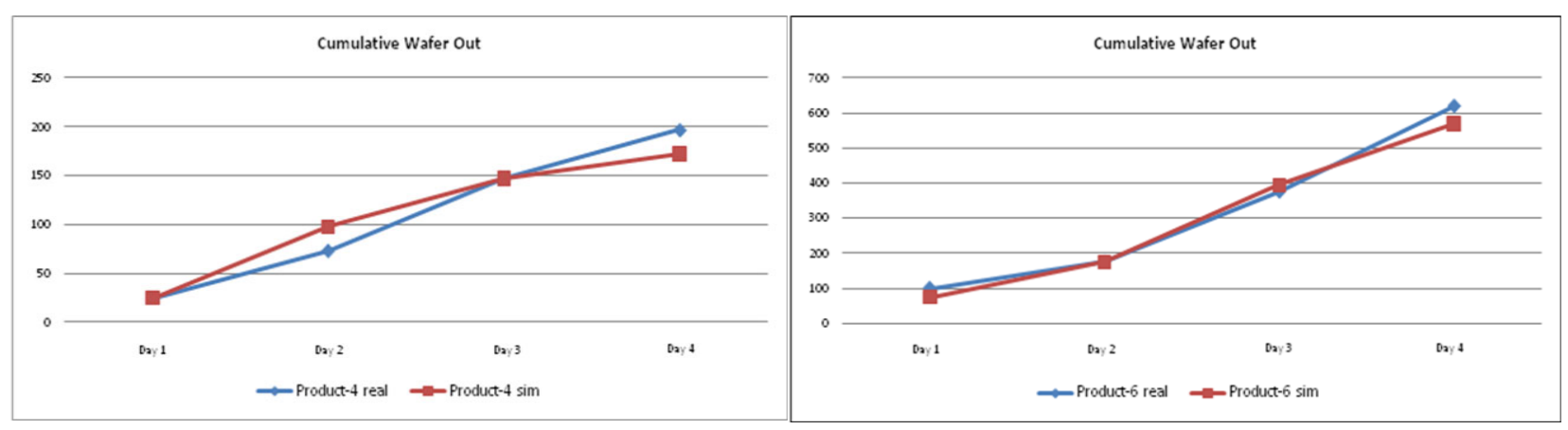

Figure 5: Cumulated Wafer Out Comparison between Simulation and Reality for a High and a Low Volume Products

\section{REFERENCES}

Bagchi, S., C. Chen-Ritzo, S.T. Shikalgar, and M. Toner. 2008. A full-factory simulator as a daily decision-support tool for $300 \mathrm{~mm}$ wafer fabrication productivity. In Proceedings of the 2008 Winter Simulation Conference, ed. S. J. Mason, R. R. Hill, L. Mönch, O. Rose, T. Jefferson, and J. W. Fowler, 2777-2782. Piscataway, New Jersey: Institute of Electrical and Electronics Engineers, Inc.

Noack D., R. Kohn, M. Mosinski, Z. Zhou, O. Rose, W. Scholl, P. Lendermann, and B. P. Gan. 2010. Data modelling for online simulation - requirements and architecture. In Proceedings of the 2010 FAIM Conference. 
Reijers, H.A., and W.M.P. v. d. Aalst. 1999. Short-term simulation: bridging the gap between operational control and strategic decision making. Proceedings of the IASTED International Conference on Modelling and Simulation.

Scholl W., 2008. Coping with typical unpredictable incidents in a logic fab. In Proceedings of the 2008 Winter Simulation Conference, ed. S. J. Mason, R. R. Hill, L. Mönch, O. Rose, T. Jefferson, and J. W. Fowler, 2030-2034. Piscataway, New Jersey: Institute of Electrical and Electronics Engineers, Inc.

\section{AUTHOR BIOGRAPHIES}

WOLFGANG SCHOLL works as a Senior Staff Expert for modelling and simulation for Infineon Technologies in Dresden (Germany). He studied Physics at the Technical University of Chemnitz (Germany) and graduated in solid-state physics in 1984. From 1984 to 1995 he worked as a process engineer for ZMD in Dresden. In 1996 he joined Infineon Technologies and initially worked on the field of capacity planning. Since 2003 he is responsible for fab simulation. His email address is <wolfgang.scholleinfineon. com>.

BOON PING GAN is the CTO of D-SIMLAB Technologies (Singapore). He has been involved in simulation technology application and development since 1995, with primary focus on developing parallel and distributed simulation technology for complex systems such as semiconductor manufacturing and port operations. He was also responsible for several operations improvement projects with wafer fabrication clients which concluded with multi-million dollar savings. He is now responsible for managing the technology and product development at D-SIMLAB as well as execution of projects in the semiconductor and engineering domains. He holds a Master of Applied Science degree, specializing in Computer Engineering. His email address is <boonping@d-simlab.com>.

DANIEL NOACK is a Research Engineer at D-SIMLAB Technologies. His focus is on simulation and simulation-based optimization. He is also a $\mathrm{PhD}$ student at Dresden University of Technology. He is a member of the scientific staff of Professor Oliver Rose at the Chair of Modelling and Simulation. He received his M.S. degree in Computer Science from Dresden University of Technology. His email address is <danieled-simlab.com>.

PATRICK PREUSS is a Software Engineer and the Deputy Manager Germany Operations of DSIMLAB Technologies (Germany). He has been working in the development of simulation-based applications for Airbus, German Aerospace Centre and Infineon with focus on heuristic optimization methods since 2005. Patrick holds a M.S. degree in computer science from Dresden University of Technology. His email address is patricked-simlab.com>.

MING LI PEH is a Product Manager at D-SIMLAB Technologies. She is responsible to execute the semiconductor product (a simulation-based optimization decision support tool) development and the delivery of product deployment at customer site. Prior to this she worked in Fuji Xerox where she was responsible for the testing and releasing of printer drivers. She holds a Bachelor of Engineering degree, specializing in Computer Engineering. Her email address is $<$ minglied-simlab.com $>$.

PETER LENDERMANN is the Co-Founder and CEO of D-SIMLAB Technologies, a Singapore-based company providing simulation-based decision support solutions and services to Aerospace, Semiconductor Manufacturing and other asset-intensive industries. Prior to this he worked at the Singapore Institute of Manufacturing Technology where he led the simulation-related research activities until spinning them off into D-SIMLAB Technologies. He has been engaged in the simulation community since the early 1990's. Peter holds a PhD in Applied High-Energy Physics from Humboldt- University in Berlin (Germany) and an MBA in International Economics and Management from SDA Bocconi in Milan (Italy). He 
is also an Adjunct Associate Professor at the Department of Industrial and Sytems Engineering at the National University of Singapore. His email address is <petered-simlab.com>.

OLIVER ROSE holds the Chair for Modelling and Simulation at the Institute of Applied Computer Science of the Dresden University of Technology, Germany. He received an M.S. degree in Applied Mathematics and a Ph.D. degree in Computer Science from Würzburg University, Germany. His research focuses on the operational modelling, analysis and material flow control of complex manufacturing facilities, in particular, semiconductor factories. He is a member of IEEE, INFORMS Simulation Society, ASIM, and GI. His web address is <www.simulation-dresden.com> and his email address is $<$ oliver.rose@tu-dresden.de>. 